\title{
EFFICACY OF FINE NEEDLE ASPIRATION CYTOLOGY, ZIEHL-NEELSEN (Z-N) STAIN AND CULTURE (BACTEC) IN DIAGNOSIS OF TUBERCULOSIS LYMPHADENITIS
}

\author{
GOSWAMI H.M. ${ }^{1}$, PARIKH U.R. ${ }^{*}$, BAROT H.P. ${ }^{1}$, VAGHELA G.M. ${ }^{2}$, YADAV K.S. ${ }^{3}$, VEGAD M.M. ${ }^{4}$ AND \\ GAZALI Z.A. ${ }^{3}$
}

1Department of Pathology, B.J. Medical College, Asarwa, Ahmedabad- 380 016, Gujarat, India. 2Department of Microbiology, Govt. Medical College, Surat- 395001, Gujarat, India.

3Padmashree Dr. D.Y. Patil Medical College and Hospital, Navi Mumbai- 400706, MS, India.

4Department of Microbiology, B.J. Medical College, Asarwa, Ahmedabad-380016, Gujarat, India.

*Corresponding Author: Email-urviparikh76@gmail.com

Received: July 26, 2012; Accepted: August 03, 2012

\section{Abstract-}

Background- Tuberculous Lymphadenitis is the commonest form of extra pulmonary tuberculosis and tissue diagnosis is the main stay in the diagnosis of extra-pulmonary tuberculosis. This study was conducted to compare cytology, $\mathrm{ZN}$ staining and culture findings of clinically suspected tuberculous lymphadenitis cases.

Methods- In the Present Study, clinically suspected cases of lymphadenopathy were undergone fine needle aspiration. The aspirates were examined cytologically followed by ZN staining and BACTEC culture.

Results- The cytology suggestive of tuberculous lymphadenitis was found in $46(76.6 \%)$ cases out of total 61 cases. Ziehl Neelsen stain demonstrated positive for acid fast bacilli (AFB) in 14 (22.9\%) cases and mycobacteria isolated from BACTEC in36 (59\%) cases. Out of 61 cases $15(24.5 \%)$ cases showed pus formation and in cytological examination 13 of those cases reported as tuberculous lymphadenitis. Aspiration mixed with blood demonstrated AFB positive in $2(5.88 \%)$ and mycobacteria were isolated in $16(48.48 \%)$ cases and cytology suggest tuberculous lymphadenitis in $21(63.63 \%)$ cases. Seven out of Nine cases of necrosis with or without neutrophils show presence of AFB. Samples having epithelioid cell granuloma with or without necrosis show AFB positive in $5(16.66 \%)$ and 2 $(25 \%)$ cases and mycobacteria isolated in $22(73.73 \%)$ and $5(62.5 \%)$ cases respectively.

Conclusion- In spite of the diagnostic piffalls, the outcomes of this study reinforce the importance of opinion i.e. Fine Needle Aspiration Cytology serves as a potent and accurate diagnostic tool forpatients presenting with Lymphadenopathy due to tuberculosis.

Key words- Fine Needle Aspiration Cytology; Ziehl-Neelsen Staining, BACTEC culture, chronic cervical lymphadenitis.

Citation: Goswami H.M., et al. (2012) Efficacy of Fine Needle Aspiration Cytology, Ziehl-Neelsen (Z-N) Stain and Culture (Bactec) in Diagnosis of Tuberculosis Lymphadenitis. International Journal of Microbiology Research, ISSN: 0975-5276 \& E-ISSN: 0975-9174, Volume 4, Issue 7, pp.-275-278.

Copyright: Copyright@2012 Goswami H.M., et al. This is an open-access article distributed under the terms of the Creative Commons Attribution License, which permits unrestricted use, distribution and reproduction in any medium, provided the original author and source are credited.

\section{Introduction}

Tubercular lymphadenitis is a common manifestation of extra pulmonary tuberculosis [1]. According to WHO tuberculosis still kills three million people every year in underdeveloped countries [2]. Tuberculosis still ravage in India even 100 years after the discovery of tubercle bacillus, with an annual incidence of 100/100,000 and a prevalence four times the incidence [3]. AIDS is one of the important causes for change in etiological profile as well as increasing cases of extra pulmonary tuberculosis [4]. Tuberculous Lym- phadenopathy is the commonest form of extra pulmonary tuberculosis in region where mycobacterial infection is highly prevalent and presents commonly in lymph nodes draining the head and neck. The conventional methods of diagnosis for tuberculosis like sputum examination of acid-fast bacilli and chest X-ray are fairly accurate in detecting the active pulmonary component of the disease. However they are not useful for detecting extra-pulmonary components [5]. By enlarge; tissue diagnosis is the mainstay in the management of cases of extra-pulmonary tuberculosis. The efficacy of 
FNAC as a diagnostic procedure is already established and it has been found to be as efficient as biopsy, particularly in cases of tubercular lymphadenitis [5]. It is very safe, trivial, cost-effective and time saving. Mycobacteria are slow growing and hence culture is not done routinely in all laboratories. Few studies have tried to correlate the cytological finding with microbiological results for the presence of acid-fast bacilli in smears and culture for mycobacteria. The current study was conducted with following objectives.

1. To determine efficacy of FNAC in detecting tuberculous lymphadenitis.

2. To evaluate the role of ZN staining and culture of aspirated material in detecting tuberculous lymphadenitis.

3. To correlate the gross appearance of aspirate and microscopic feature of lymph node aspirate with AFB positivity and culture.

\section{Material and Methods}

A retrospective study of forty cases over a period of one year i.e. from January, 2011 to December, 2011 was carried out to determine the diagnostic accuracy of FNAC for lymphadenopathy in suspected tuberculous patients, in pathology department of our tertiary care hospital. The patients were examined clinically and after detailed clinical history and physical examination, routine hemogram with ESR; aspiration was carried out using 22 gauge needle and standard precautions. The material obtained was used for cytological examination, ZN smears and BACTEC culture, also the gross appearance of aspirate was noticed as either purulent or cheesy or blood mixed. Smears were immediately fixed in $95 \%$ ethyl alcohol as well as some are kept unfixed. Unfixed smears were stained with May Graunwald Giemsa (MGG) while fixed smears were stained with Haematoxyline and Eosin stain (H\&E) and Papanicolaou (PAP) stain. Cytopathological diagnosis had been recorded in each case. Special stains used were Ziehl Neelson (ZN) stain for detecting acid fast bacilli (AFB). Remaining material was inoculated in BACTEC (Middle brook $7 \mathrm{H} 12 \mathrm{~B}$ ) vial taking care to have at least $0.5 \mathrm{ml}$ volume of test material in the vial. A record was made of all relevant findings including the gross appearance of the aspirate. The gross appearance of aspirate was described as caseous for cheese like or yellow-white aspirate and blood mixed when material was haemorrhagic. Cytology smears and ZN stain smears were examined in cytology section. BACTEC vial containing aspirated material was sent to microbiology laboratory of our hospital without delay where first medium was supplemented with mixture of anti-microbial called PANTA, which contains polymyxin $-B$, amphotericin- $B$, nalidixic acid, trimethoprim and azlocillin to reduce the contamination. Then initial reading Growth Index (GI) was taken and then incubated at $37^{\circ} \mathrm{C}$. Readings of $\mathrm{GI}$ were taken on day $1,3,5,7,9,12$ for first 15 days and then weekly upto 45 days. The diagnosis of tuberculous lymphadenitis was made when the following criteria's were met: the presence of epithelioid cell granuloma with or without necrosis and/or ZN smear positivity for Acid-Fast Bacilli (AFB) and/or positive culture for mycobacteria. Data was recorded and statistically analyzed using chi square. Specificity, sensitivity, Positive Predictive Value (PPV), Negative Predictive Value (NPV) and likelihood ratio of smear and cytology were compared. In our study, we have complemented FNAC with ZN staining and culture.

\section{Results}

A total of forty patients with peripheral lymphadenopathy diagnosed and treated at our tertiary care hospital over a period of twelve months i.e. from January, 2011 to December, 2011 were taken into consideration. In all the cases, pre-operative FNAC was done and diagnosis was recorded. Age wise distribution was noted between 5-67 years during the present study. Our youngest patient was 5 year female while the oldest patient was 63 year male. Most of the cases were found in 31-40 years of age (37.5\%) followed by 21-30 years $(22.5 \%)$. Out of 40 cases studied, 22 patients were male while 18 patients were female.

\begin{tabular}{|ccccc|}
\multicolumn{5}{c}{ Table 1- } \\
Age (Years) & Male & Female & Total & Percentage \\
$0-10$ & - & 1 & 1 & 2.5 \\
$11-20$ & 4 & 4 & 8 & 20 \\
$21-30$ & 4 & 5 & 9 & 22.5 \\
$31-40$ & 9 & 6 & 15 & 37.5 \\
$41-50$ & 2 & 2 & 4 & 10 \\
$>50$ & 3 & - & 3 & 7.5 \\
TOTAL & 22 & 18 & 40 & \\
\hline
\end{tabular}

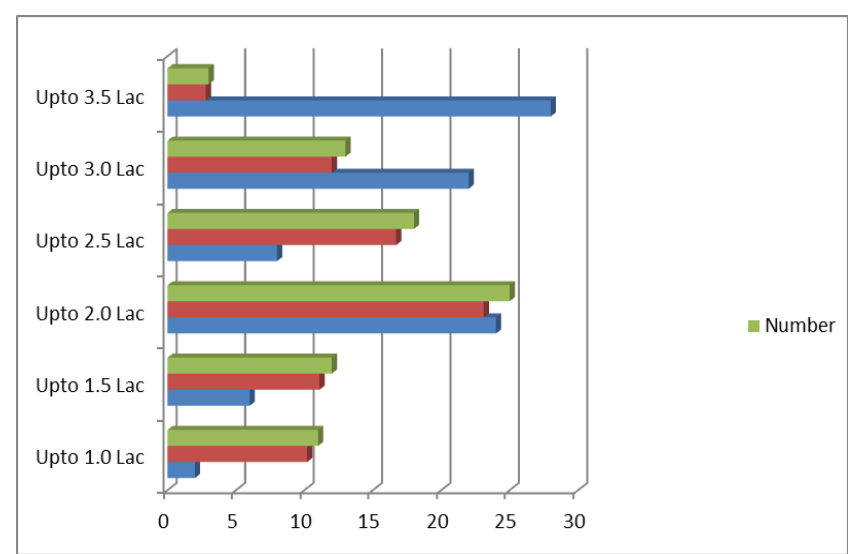

Chart 1- A chart showing age wise correlation with male is to female distribution

Most of the cases were found in 31-40 years of age (37.5\%) followed by $21-30$ years $(22.5 \%)$. Out of 40 cases studied, 22 patients were male while 18 patients were female.

Thus the peripheral lymphadenopathy showed male predominance - with the male to female ratio 1.22:1. The most common site of peripheral lymphadenopathy was the cervical group; consist of 31 out of 40 cases $(77.5 \%)$. FNAC smears of all 40 patients were examined, followed by ZN staining and BACTEC culture. Minimum incubation time for isolation of mycobacteria through BACTEC culture was 14 days and maximum was 52 days (mean 24 days) as per the SOP.

Table 2-

\begin{tabular}{|llll|} 
Sr. No. Lesions Encountered & $\begin{array}{l}\text { Number of } \\
\text { Cases }\end{array}$ & $\begin{array}{l}\text { Percentage } \\
(\%)\end{array}$ \\
\hline 1 & Tuberculous Abscess & 24 & 60 \\
2 & Acute Suppurative Abscess & 5 & 12.5 \\
3 & Chronic Non-specific Lymphadenitis & 11 & 27.5 \\
& TOTAL & 40 & 100 \\
\hline
\end{tabular}

During cytological examination; tuberculous lymphadenopathy (60 $\%$ ) was the most common lesion encountered during the present 
study (24 out of 40 patients). Out of them, 12 patients had caseous necrosis and granuloma, 7 patients had granuloma without caseous necrosis and 5 patients had caseous necrosis without granuloma formation.

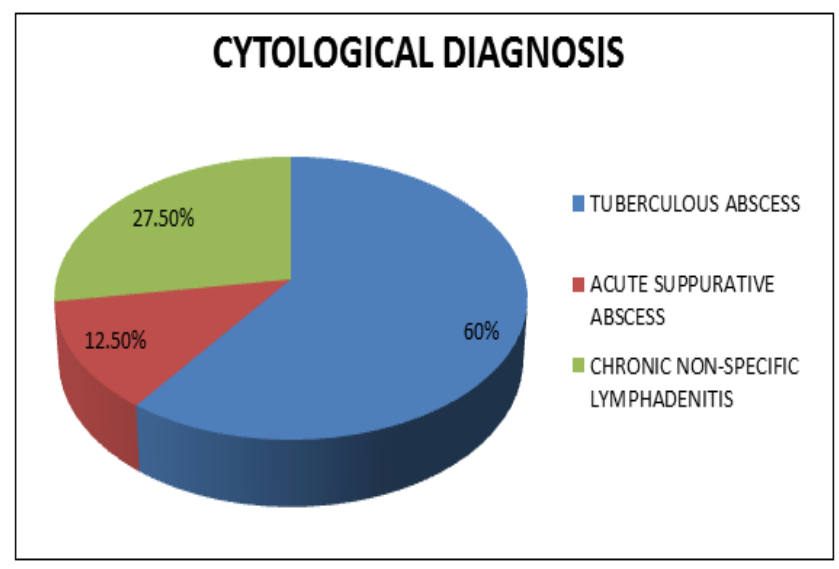

Chart 2- Pie diagram showing cytological diagnosis in lymphadenopathy

During cytological examination; tuberculous lymphadenopathy (60 $\%)$ was the most common lesion encountered during the present study (24 out of 40 patients). Out of them, 12 patients had caseous necrosis and granuloma, 7 patients had granuloma without caseous necrosis and 5 patients had caseous necrosis without granuloma formation.

All the patients underwent AFB stain and culture examination by BACTAC. Thirteen patients (32.5\%) showed positive AFB staining and BACTEC isolated mycobacteria in 18(45\%) cases.

Table 3-

\begin{tabular}{lllll|}
$\begin{array}{llll}\text { Gross Appearance } \\
\text { Of Aspirate }\end{array}$ & Cases (\%) & $\begin{array}{l}\text { Cytology S/O } \\
\text { TBLN (\%) }\end{array}$ & $\begin{array}{l}\text { AFB +Ve on } \\
\text { ZN Stain (\%) }\end{array}$ & $\begin{array}{l}\text { Culture Isolated } \\
\text { Mycobacteria (\%) }\end{array}$ \\
\hline BLOOD MIXED & $19(47.5 \%)$ & $7(36.84 \%)$ & $5(26.32 \%)$ & $9(47.37 \%)$ \\
CHEESY & $9(22.5 \%)$ & $9(100 \%)$ & $4(44.44 \%)$ & $3(33.33 \%)$ \\
PURULENT & $12(30 \%)$ & $8(66.67 \%)$ & $4(33.33 \%)$ & $6(50 \%)$ \\
TOTAL & 40 & 24 & 13 & 18 \\
\hline
\end{tabular}

Table 4-

\begin{tabular}{|c|c|c|c|}
\hline Morphology on Cytology & Cases(\%) & $\begin{array}{l}\text { AFB } \\
\text { Positive (\%) }\end{array}$ & $\begin{array}{l}\text { Culture } \\
\text { Positive (\%) }\end{array}$ \\
\hline Epith & $7(2$ & $4(57.14 \%)$ & $4(57.14 \%)$ \\
\hline Epithelioid cell granuloma with necrosis & $12(50 \%)$ & $5(41.67 \%)$ & $9(75 \%)$ \\
\hline Necrosis without granuloma & $5(20.83 \%)$ & $4(80 \%)$ & $5(100 \%)$ \\
\hline Total & 24 & 13 & 18 \\
\hline
\end{tabular}

\section{Discussion}

Although histopathology is most rewarding for diagnosis of cervical lymphadenitis, its feasibility is limited due to non-acceptability, being an invasive procedure. Previously, biopsy was used for diagnosis of tubercular lymphadenitis; now it has been greatly replaced by FNAC. The correlation of cytopathology and histopathology has already been discussed by several researchers [6-9]. In literature, so far, there are only a few reports available for simultaneous FNAC and culture used for the diagnosis of tuberculosis. Purohit, et al have used Mantoux test, skiagram chest, histopathology and culture of mycobacteria as diagnostic parameters [10].

The present study consists of 40 clinically suspected cases of tu- berculous lymphadenitis with a Male/Female ratio of 1.22:1 and in the age group of 5 to 67 years, who attended the outpatient department of our tertiary care hospital. Similar results were obtained by Mistri et al, the male:female ratio is 1.25:1; with the youngest patient was 1 year old [2]. In our study, we have complemented FNAC with ZN staining and culture altogether. The identification of species of mycobacteria would help to study various biological properties of mycobacteria including drug sensitivity and therapeutic approach. The results obtained were in the range that was observed by other authors, who carried out same procedure.

The results obtained were in the range that was observed by other authors, who carried out same procedure. When gross appearance of aspirate was correlates with AFB and culture positivity, maximum positivity was observed in cases with purulent aspirate for both [2]. On cytomorphological correlation maximum positivity for AFB $(80 \%)$ and culture (100\%) was found in smears showing necrosis without epithelioid granuloma. Similar results were obtained by Mistry et al [2]. The overall ZN staining positivity for AFB was $32.5 \%$ and in $40 \%$ cases mycobacteria were isolated by culture during our study. Patwardhan noted that AFB culture positivity have sensitivity of $74.5 \%$ and specificity of $100 \%$ while Tyagi et al. have reported sensitivity of $23 \%$ in their study In all the culture, positivity was significantly higher than ZN smear positivity ( $p$ value $=0.00005 \%)[11,12]$.

When culture was taken as the reference method, the outcomes of cytological examination were as follows: Sensitivity is $97.2 \%$ Specificity is $56 \%$ and Accuracy is $80.32 \%$. Thus, the Sensitivity of FNAC was higher and diagnostic accuracy was comparable with other studies.

The diagnostic difficulties encountered were parallel to those experienced by different authors working on similar topic, a case in point being false negative cytology diagnosis in case with purulent aspirate which calls for ZN staining in every case suspected of tuberculous in origin [5].

\section{Conclusion}

In spite of the diagnostic pitfalls, the results obtained on analytical examination of the study carried out reinforce the opinion that fine needle aspiration cytology serves as a potent and accurate diagnostic tool for patients presenting with lymphadenopathy due to tuberculosis. This approach carries numerous advantages, the principal ones being enhanced patient compliance obtainable samples, easily aspirated tissue and an inexpensive yet accurate outcome. Another essential and practical feature includes the staining of aspirated smears by Ziehl-Neelsen technique especially in purulent material for an improved diagnostic accuracy. These procedures facilitate a reduction in operative morbidity as well as the time span for a definitive diagnosis. Finally, culture methods for the specific mycobacteria proved to be the mainstay in the diagnosis of tuberculosis. In conclusion, FNAC serves as one of the most accurate frontline method for the diagnosis of tuberculous lymphadenitis. It is more than adequately supplemented by the mandatory Ziehl-Neelsen technique carried out on smears as well as culture methods which can be relied upon in conjunction with the above procedures. However, newer diagnostic techniques would be more predictive in improving the diagnosis of tuberculous lymphadenitis than conventional methods. 


\section{References}

[1] Gadre D.V., Singh U.R., Saxena K., Bhatia A. and Talwar V. (1991) Ind. J. Tub, 38, 25-27.

[2] Mistry Y., Ninama G.N., Mistry K., Rajat R., Parmar R. and Godhani A. (2012) National Journal of Medical Research, 2(1), 77-80.

[3] Battacharya S. et al. (1998) Indian Jr. of Medical Sci., 52, 498506.

[4] MPS Menon (1997) Indian Journal of Tuberculosis, 44, 3-9.

[5] Singh J.P. et al. (1989) Indian Journal of Patho. Microbio., 32, 101-104.

[6] Nagpal B.L., Dhar C.N., Singh A. and Bahi H.H. (1982) Ind. J. P.M., 25, 35.

[7] Patra A.K., Nanda B.K., Mohaptra B.K. and Panda A.K. (1983) IJPM, 26, 273.

[8] Dandapat M.C., Panda B.K., Patra A.K. and Acharya N. (1987) Ind. J. Tub., 34,139.

[9] Tripathy S.N., Mishra N., Patel N.M., Samantray D.K., Das B.K. and Mania R.N. (1985) Ind. J. Tub., 32, 130.

[10]Purohit S.D., Gupta M.L., Sarkar S.K., Gupta P.R. and Tanwar Dilip Jain (1987) Ind. J. Tub., 34, 22.

[11]Patwardhan S.A., Bhargava P., Bhide V.M. and Kelkar D.S. (2011) Ind. J. Medical Microbiology, 29(4), 389-39.

[12]Singh K.K., Tyagi J.S., Muralidhar M., Kumar A., Chattopadhyaya T.K., Kapila K. et al. (2000) J. Clin. Pathol., 53, 355-61. 